\begin{tabular}{|c|c|}
\hline Title & Nanometer site analysis of electron tracks and dose local ization in bio-cells exposed to X-ray irradiation \\
\hline Author(s) & Date, H.; Y oshii, Y .; Sutherland, K.L. \\
\hline Citation & $\begin{array}{l}\text { Nuclear Instruments and Methods in Physics Research. Section B, Beam Interactions with Materials and A toms, 267(7), } \\
\text { 1135-1138 } \\
\text { https://doi.org/10.1016/.nimb.2009.02.048 }\end{array}$ \\
\hline Issue Date & $2009-04-15$ \\
\hline Doc URL & http:/hdl.handle.net/2115/38462 \\
\hline Type & article (author version) \\
\hline File Information & NIM_B267_Date.pdf \\
\hline
\end{tabular}

Instructions for use 


\title{
Nanometer site analysis of electron tracks and dose localization in bio-cells exposed to $\mathrm{X}$-ray irradiation
}

\author{
H. Date*, Y. Yoshii ${ }^{1}$, K.L. Sutherland ${ }^{2}$ \\ Faculty of Health Sciences, Hokkaido University, Sapporo 060-0812, Japan \\ ${ }^{1}$ School of Medicine, Hokkaido University, Sapporo 060-0812, Japan \\ ${ }^{2}$ Graduate School of Medicine, Hokkaido University, Sapporo 060-8638, Japan
}

\begin{abstract}
This paper reports on a Monte Carlo simulation analysis of the ionization and excitation clusters in electron tracks, which may contribute to radiation damage of bio-cells with high probabilities. The study is aimed to investigate the energy transfer to the cell nucleus exposed to X-rays from low dose in the environment to high dose in radiation therapy. As an example, we adopted a water phantom (rectangular solid, $30 \times 30 \times 30 \mathrm{~cm}$ ) exposed to a linac $6 \mathrm{MV}$ X-ray to calculate the expected energy transfer to electrons in liquid water. Then, a track simulation of the electrons produced by the photon interactions was performed. In the track simulation, the behavior of low energy electrons below $1 \mathrm{keV}$ is of particular importance to form clusters of ionization and excitation events within a nanometer scale ( $<100 \mathrm{~nm})$. We describe the clustering pattern in terms of aggregation index (AI), the distribution of point to point distance below several nanometers between the events. By comparing the distribution with that of the Poisson configuration, the clustering effects of the events including successive radical processes are evaluated.
\end{abstract}




\section{Keywords}

Electron track, Monte Carlo simulation, dose localization, ionization and excitation events

PACS numbers: $34.80 . \mathrm{Dp}$

*Corresponding author: H. Date

Postal address: N12-W5, Kita-ku, Sapporo 060-0812, JAPAN

Email address: date@hs.hokudai.ac.jp

Tel: +81-11-706-3423, Fax: +81-11-706-4916 


\section{Introduction}

Ionization and excitation of atoms and molecules in bio-tissues take place abundantly following irradiation due to inelastic collisions of the electrons produced by primary incident particles. This is true if the primary particles are "low LET radiations" such as X-rays, $\gamma$-rays, and protons with high energies above several tens of MeV. From the microscopic point of view, therefore, the electron track process is essential in low LET irradiations for transferring energy to the materials rather than the primary radiation interaction itself. A review of recent progress in the investigation of the electron track structure has been provided by Nikjoo et al (2006) [1] to show the importance of such a process. The ionization and excitation events are inclined to occur densely by low energy $(<1 \mathrm{keV})$ electron collisions along various local traces of the electron forming spurs or blobs. This is called ionization and excitation “clusters” that may lead to damage of DNA and other elements of bio-materials with high probabilities [-1-4].

In this study, we have performed Monte Carlo simulations for photon, electron and radicals to investigate spatial concentration of the energy transfer processes through electron collisions in liquid water on the assumption that an equivalent condition for electron track holds when inside of bio-cells. In order to evaluate the concentration degree of events with clusters, we have introduced "aggregation index (AI)" that is the distribution of distance between two arbitrary event locations below several nm. The AI enables us to evaluate the probability to cause damage due to adjacent plural reactions such as double strand breaks (DSBs). Diffusion processes of radicals starting at the ionization and excitation positions are also discussed along the lines of the AI consideration.

\section{Simulation model and method}

For the present simulation, we adopted a model condition with a water phantom for calibrating dosimeters, commonly used in radiation therapy. First, a Monte Carlo simulation 
of linac $6 \mathrm{MV}$ X-ray photons incident on a water phantom (rectangular solid, typically $30 \times 30 \times 30 \mathrm{~cm}$ ) was performed to calculate energy transfer to electrons through photon interaction. The radiation intensity was set to yield the absorbed dose from 0.1 Gy to $6.0 \mathrm{~Gy}$. The energy and ejection angle of electrons were sampled in a cube $1 \mathrm{~cm}$ on a side located at $10 \mathrm{~cm}$ depth from the surface center of the phantom, where the field size is $10 \times 10 \mathrm{~cm}$ for parallel X-ray beam irradiation. Second, we made a Monte Carlo track simulation of the electrons produced in the photon interactions. The electron track was followed with an event-by-event algorithm using a set of electron collision cross sections for liquid water $[5,6]$ and the scattering angle distribution for elastic and ionization collisions [7-9]. To reduce the computation time for vast amount data of ionization and excitation positions, a cyclic boundary condition for a cube voxel $100 \mu \mathrm{m}$ on a side was applied in this simulation. Then, we evaluated the concentration degree of the events (clustering) by counting the point to point distance distribution within a sphere $10 \mu \mathrm{m}$ in diameter imitating a cell-nucleus inside the voxel.

The simulation codes used here have been developed by the authors. In the photon code, the cross sections for the photon interactions (i.e., coherent scattering, photoelectric effect, Compton scattering and pair production) are taken from Storm and Israel (1970) [10], and algorithms and related data by several papers [11-14] are used. Particular attention was paid to the electron track code because the behavior of electrons with low energies below several hundred $\mathrm{eV}$ has some uncertainty among the codes reported depending on the electron collision cross sections built in the code [1,4,15-18]. Comparisons of the CSDA range and stopping power deduced by our code with those in ICRU Report 37 [19] were made as benchmark tests, and we confirmed good agreements between those for the both quantities, respectively, in the electron energy ranging from $10 \mathrm{eV}$ to $10 \mathrm{MeV}$. In the radical simulation, radical diffusion length in a random direction was estimated from the diffusion equation as [20] 


$$
\lambda=\sqrt{6 D t}
$$

where $D$ is the diffusion coefficient and $\lambda$ is the diffusion length at an elapsed time $t$. For a trial example to estimate distance distribution between two radicals, the $\mathrm{OH}$ radical was adopted and its diffusion coefficient was assumed to be $2.8 \times 10^{-5} \mathrm{~cm}^{2} / \mathrm{s}$ [21]. The time step for chasing the diffusion process $\Delta \mathrm{t}=10^{-12}$ sec was set in the present study.

\section{Results and discussion}

The energy distribution of electrons generated by $6 \mathrm{MV}$ X-ray interactions in the water phantom is shown in Fig.1. A typical spectrum of $6 \mathrm{MV}$ X-ray used here is also plotted in Fig.1, which is obtained from a calculation by Schiff's formula [22] for photon production at the Tungsten target and a consideration of beam attenuation by a flattening filter (Tungsten, $12.5 \mathrm{~mm}$ thickness) after the production [23]. As shown in Fig.1, the energy of electrons arising from the photon interactions distributes abundantly below $1 \mathrm{MeV}$ (average 0.568 $\mathrm{MeV}$ ), and most of the electrons are from Compton scattering. This result implies that the average number of photon interactions to yield 2 Gy (typical absorbed dose in radiation therapy per fraction) in the phantom is roughly $2.2 \times 10^{10} \mathrm{~cm}^{-3}$, and the average number per cell nucleus is 11.5 for the nuclear diameter of $10 \mu \mathrm{m}$. The number of electron tracks arising from the photon interactions per cell nucleus is well described by the Poisson distribution for this average value.

Figure 2 shows the distribution of distance between inelastic collision processes (i.e., ionization and excitation). The distribution curve for Poisson configuration is also plotted for comparison. Here, the Poisson configuration stands for the random point formation in a bounded area. When we assume a sphere volume of radius $\mathrm{R}$, the probability distribution of distance $x$, between two arbitrary points in the sphere is described by

$$
F(x)=\frac{3}{16 R^{6}} x^{5}-\frac{9}{4 R^{4}} x^{3}+\frac{3}{R^{3}} x^{2}, \quad(0 \leq x \leq 2 R)
$$


see [24,25]. As is shown in Fig.2, the curve for the electron track has a local maximum at around 3-4nm, while the curve coincides with that of the Poisson configuration greater than about $100 \mathrm{~nm}$. We call this distribution within a short distance (below several tens of $\mathrm{nm}$ ) “aggregation index (AI)" of the inelastic collision events. The local maximum is attributable largely to the clustering of ionization and excitation events. Ionization and excitation occur significantly with low energy electrons slowed down from $1 \mathrm{keV}$ to $10 \mathrm{eV}$ forming the clusters, independent of the initial energy [4,5]. Two neighboring events (of ionization and/or excitation) and succeeding radical formations in a few nanometers must be fundamental for causing double strand breaks (DSBs) of DNA since this distance scale is equivalent to the double helix local site. The curve in the short distance region seems to be shifted to upper values linearly as dose increases. In Fig.3(a), the integrated value over $0-10 \mathrm{~nm}$ is plotted as a function of dose for three cases, ionization and excitation events, ionization events only and excitation events only, respectively. Here, $10 \mathrm{~nm}$ was assumed in the integration as the upper limit for possible length causing DSBs. This value may be rather large, but the subsequent discussion stays unchanged granting that the limit is set to $3.4 \mathrm{~nm}$ (equivalent to the length of 10 base pairs for DNA [26]). In our calculation range up to $6 \mathrm{~Gy}$, the integrated value is simply proportional to dose. This result suggests that the inter-track damage (associated with two adjacent tracks shown in Fig.3(b)) for directly inducing DSBs of DNA hardly occurs. In other words, it can be interpreted that the two arbitrary tracks are found in space independently. If the inter-track processes have risen up in a local site within $10 \mathrm{~nm}$, the curve in Fig.3(a) should have been hyper-linear with dose increment.

Apart from the possibility to attack molecules directly by electrons, the indirect reactions by radicals were examined by the radical simulation. Radical species such as $\mathrm{OH}, \mathrm{e}_{\mathrm{aq}}^{-}$ (aqueous electron), $\mathrm{H}_{3} \mathrm{O}^{+}$and $\mathrm{H}$ were taken into account. The thermal diffusion coefficients for each radical have been given in pure water [20,21]. As a typical radical having a minimum value of diffusion coefficient among the above species, we adopted $\mathrm{OH}$ radical to 
see the diffusion behavior. Figure 4 shows the AI for $\mathrm{OH}$ radical formation originating from the electron tracks for initial electron energies, $10 \mathrm{keV}$ and $1 \mathrm{keV}$, as a function of time. Here, the radical production rate was assumed to be one $\mathrm{OH}$ radical per event (ionization or excitation) at each event's location. The results for both cases show that two arbitrary $\mathrm{OH}$ radicals produced along the electron track spread out from each other more than $10 \mathrm{~nm}$ by diffusion at least in $0.1 \mu \mathrm{s}$, leading to the Poisson configuration in the lifetime of the radical ( $1 \mu \mathrm{s})$. The results in Fig.4 have statistical variations because these computations are subject to one track history. However, the trials for some histories showed the same tendency in regard to the time dependence. The result of this radical spatial formation may imply that the possibility of the inter-track damage of DNA is negligible even though the radical processes are taken into account. A problematic issue of this simulation is the validity of the diffusion coefficient because the value used here is for pure water while the real situation of radicals is in bound (by hydrogen bonding) water with a variety of proteins and other materials. It is well known that the mobility of radicals is much impaired in the bound water by some orders of magnitude [27]. Therefore, further consideration is necessary for the radical diffusion in the real cell condition filled with bound water.

The present study is based upon the assumption of a linac 6 MV X-ray irradiation with rather high dose. However, it should be noted that the AI approach and the radical behavior from one electron track are applicable to a wide range of dose.

\section{Conclusion}

In this study, we have carried out Monte Carlo simulations for photon, electron and radicals assuming a water phantom model exposed to $6 \mathrm{MV}$ X-ray irradiation. The results are summarized as follows.

(1) The ionization and excitation clusters were evaluated by the distance distribution between the events, and the distribution was compared with that of Poisson configuration. The 
difference between them is significant in a short distance below a few tens of nm showing a major possibility to cause double strand breaks (DSBs) of DNA. The integrated value over 0-10 $\mathrm{nm}$ of this distribution is almost linear as a function of dose up to 6 Gy. This linearity suggests that the inter-track damage in DNA scale sites hardly occurs through the electron direct collisions even with the therapeutic dose.

(2) The radical simulation (typically with $\mathrm{OH}$ radical) was made starting at the ionization and excitation locations of the electron track. The distance distribution between two arbitrary $\mathrm{OH}$ radicals spreads out $(>10 \mathrm{~nm})$ from each other in a short period of time $(0.1 \mu \mathrm{s})$ converging to the Poisson configuration.

\section{Acknowledgements}

This work was financially supported in part by the Grant-in-Aid for Scientific Research of Japan.

\section{References}

[1] H. Nikjoo, S. Uehara, D. Emfietzoglou, F.A. Cucinotta, Track-structure codes in radiation research, Radiat. Measurements 41(2006) 1052-1074

[2] D.T. Goodhead, H.P. Leenhouts, H.G. Paretzke, M. Terrissol, H. Nikjoo and R. Blaauboer, Track structure approaches to the interpretation of radiation effects on DNA, Radiat. Prot. Dos. 52 (1994) 217-223

[3] B. Grosswendt, Formation of ionization clusters in nanometric structures of propane-based tissue-equivalent gas or liquid water by electrons and $\alpha$-particles, Radiat. Environ. Biophys. 41 (2002) 103-112

[4] H.G. Paretzke, D.T. Goodhead, L.G. Kaplan, M. Terrissol, Atomic and molecular data for radiotherapy and radiation research, Chapter 9: Track structure quantities, IAEA -TECDOC -799 (1995)

[5] H. Date, K.L. Sutherland, H. Hasegawa, and M. Shimozuma, Ionization and excitation 
collision processes of electrons in liquid water, Nucl. Instr. and Meth. B 265 (2007) 515-520

[6] D. Emfietzoglou, Inelastic cross-sections for electron transport in liquid water: a comparison of dielectric models, Radiat. Phys. Chem. 66 (2003) 373-385

[7] D.J. Brenner and M. Zaider, A computationally convenient parameterization of experimental angular distributions of low energy electrons elastically scattered off water vapour, Phys. Med. Biol. 29(4) (1983) 443-447

[8] B. Grosswendt and E. Waibel, Transport of low energy electrons in nitrogen and air, Nucl. Instr. and Meth. 155 (1978) 145-156

[9] A.E.S. Green and T. Sawada, Ionization cross sections and secondary electron distributions, J. Atmosph. Terr. Phys. 34 (1972) 1719-1728

[10] E. Storm and H.I. Israel, Photon Cross Sections from $1 \mathrm{keV}$ to $100 \mathrm{MeV}$ for Elements Z=1 to Z=100, Nuclear Data Tables A7 (1970) 565-681

[11] D.E. Reaside, Monte Carlo Principles and Applications, Phys. Med. Biol. 21 (1976) 181-197

[12] C.D. Zerby, A Monte Carlo Calculation of the Response of Gamma-Ray Scintillation Counters, Methods in Computational Physics, Vol.1, Ed. B. Alder, S. Fernback and M. Rotenberg, Academic Press, New York (1963) 89-134

[13] J.H. Hubbell et al, Atomic Form Factors, Incoherent Scattering Functions and Photon Scattering Cross Sections, J. Phys. Chem, Ref. Data 4 (1975) 471-538

[14] G. Gaggero, Monte Carlo Calculations for the Photofractions and Energy Loss Spectra of Ge(Li) Semiconductor Detector, Nucl. Instr. and Meth. 94 (1971) 481-492

[15] H.G. Paretzke, J.E. Turner, R.N. Hamm, H.A. Wright, and R.H. Ritchie, Calculated yields and fluctuations for electron degradation in liquid water and water vapor, J. Chem. Phys. 84(6) (1986) 3183-3188

[16] M. Dingfelder, D. Hantke, M. Inokuti, H.G. Paretzke, Electron inelastic-scattering cross sections in liquid water, Radiat. Phys. Chem. 53 (1998) 1-18

[17] D. Emfietzoglou, K. Karava, G. Papamichael and M. Moscovitch, Monte Carlo 
simulation of the energy loss of low-energy electrons in liquid water, Phys. Med. Biol. 48 (2003) 2355-2371

[18] D. Emfietzoglou, F.A. Cucinotta, H. Nikjoo, A Complete Dielectric Response Model for Liquid Water: A Solution of the Bethe Ridge Problem, Radiat. Res. 164 (2005) 202-211

[19] ICRU Report 37, Stopping powers of electrons and positrons, International Commission on Radiation Units and Measurements, Washington DC, Vol.37 (1984)

[20] J.E. Turner, “Atoms, radiation, and radiation protection”, John Wiley \& Sons, Inc., New York, $\quad 2^{\text {nd }}$ Ed. (1995)

[21] S.Uehara and H. Nikjoo, Monte Carlo Simulation of Water Radiolysis for Low-energy Charged particles, J. Radiat. Res. 47 (2006) 69-81

[22] L.I. Schiff, Energy angle distribution of thin target bremsstrahlung, Phys. Rev. 83 (1951) $252-253$

[23] H. Kato, Air Kerma Transmission Factors of Scattered X-rays in the Maze of a Liniac Room for Lead Shield, J. J. Radiol. Tech. (in Japanese) 61 (2004) 96-103

[24] T. Radivoyevitch, S. Kozubek, and R.K. Sachs, Biologically based risk estimation for radiation-induced CML Inferences from BCR and ABL geometric distributions, Radiat. Environ. Biophys. 40 (2001) 1-9

[25] A.M. Kellerer, and H.H. Rossi, A generalized formulation of dual radiation action, Radiat. Res. 75 (1978) 471-488

[26] H.Nikjoo, P. O’Neill, D.T. Goodhead, M. Trerrissol, Computational modelling of low-energy electron-induced DNA damage by early physical and chemical events, Int. J. Radiat. Biol. 71 (1997) 467-483

[27] B. Aydogan, D.T. Marshall, S.G. Swarts, J.E. Turner, A.J. Boone, N.G. Richards, and W.E. Bolch, Site-Specific OH Attack to the Sugar Moiety of DNA: A Comparison of Experimental Data and Computational Simulation, Radiat. Res. 157 (2002) 38-44 


\section{Figure captions}

\section{Figure 1.}

Energy distribution of electrons produced by a linac 6 MV X- ray.

\section{Figure 2.}

Distribution of distances between ionization and excitation events in comparison with that of Poisson configuration.

\section{Figure 3.}

(a) Distance frequency integrated from 0 to $10 \mathrm{~nm}$ as a function of dose:

$\checkmark$ both events for ionization and excitation, $-\square$ ionization events only, $\prec$ excitation events only.

(b) Basic concept of intra-track and inter-track processes in the DNA scale.

\section{Figure 4.}

Aggregation index (AI) for $\mathrm{OH}$ radical diffusing as a function of time: (a) for $10 \mathrm{keV}$ electron track and (b) for $1 \mathrm{keV}$ electron track. 


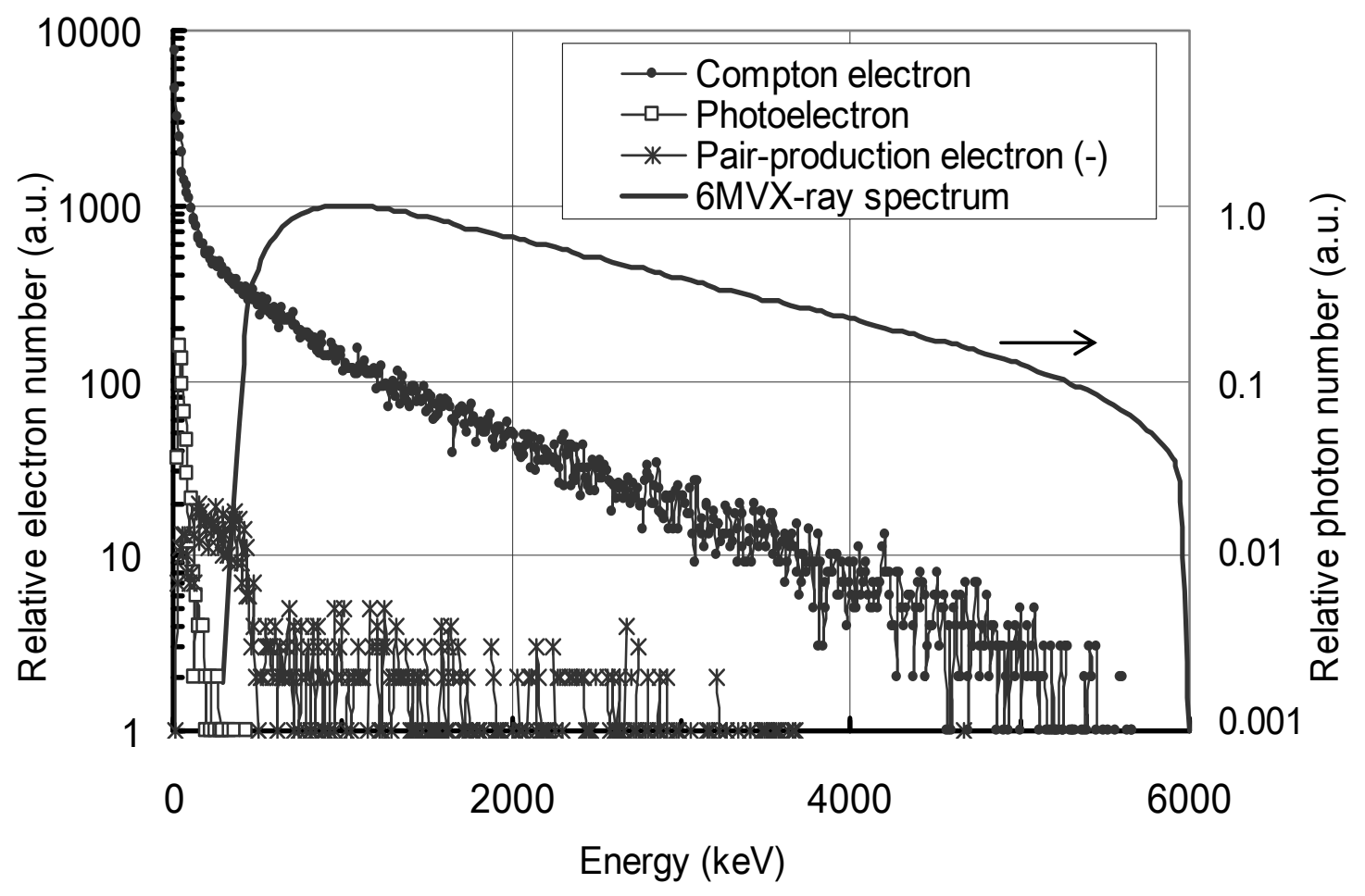

Figure 1.

Date et al. 


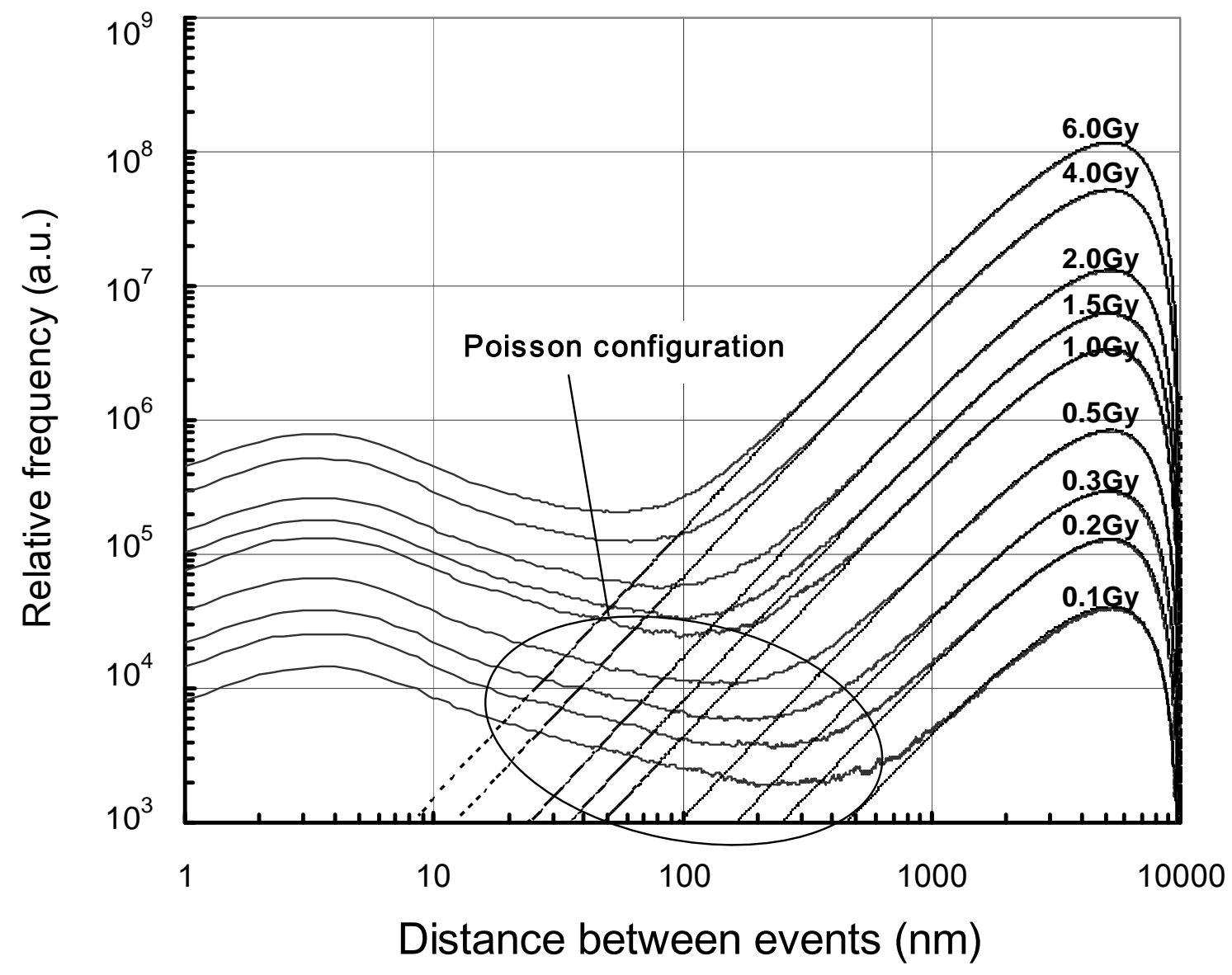

Figure 2.

Date et al. 


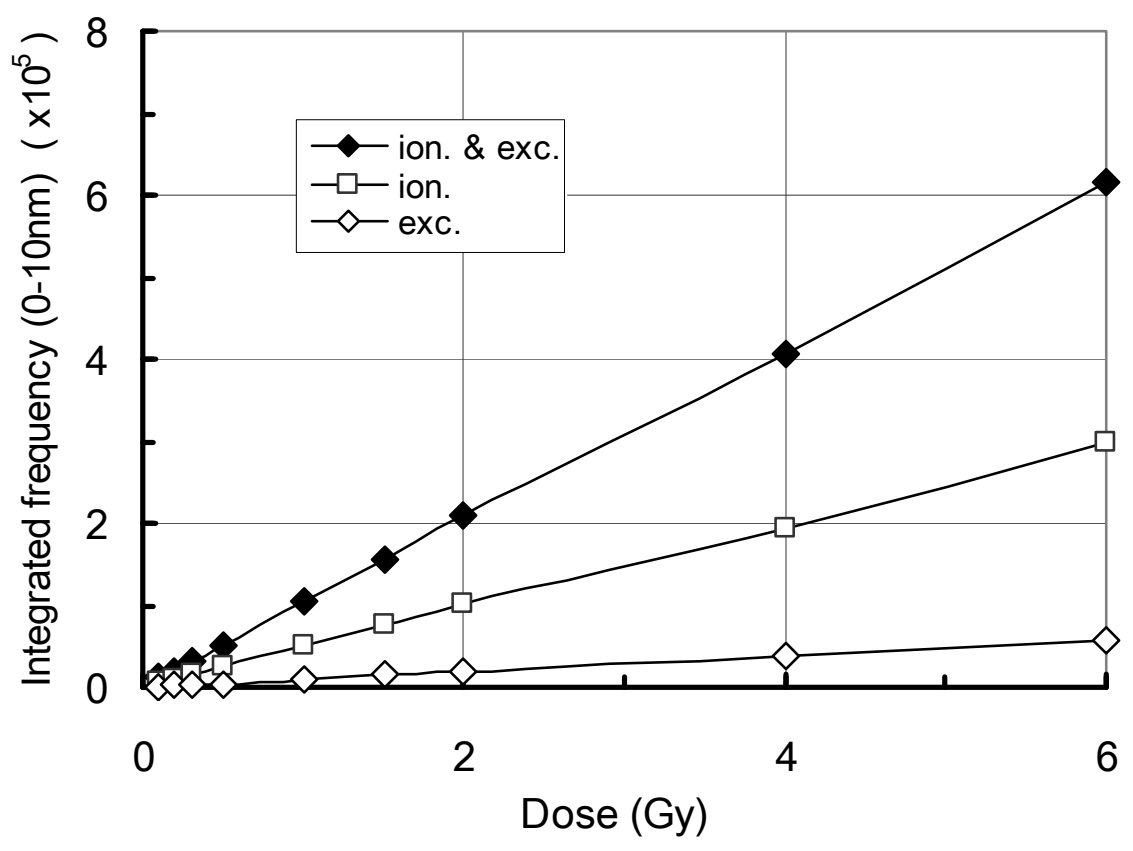

(a)

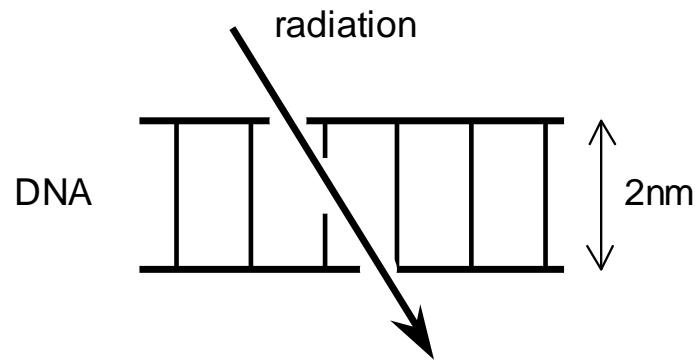

(intra-track process)

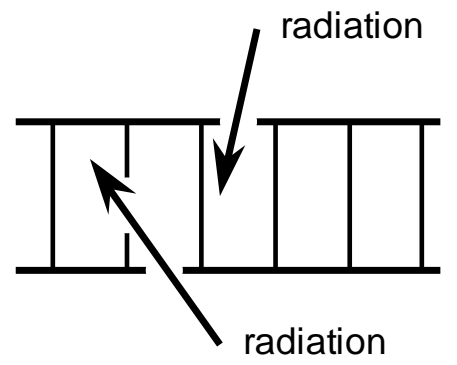

(inter-track process)

(b)

Figure 3.

Date et al. 


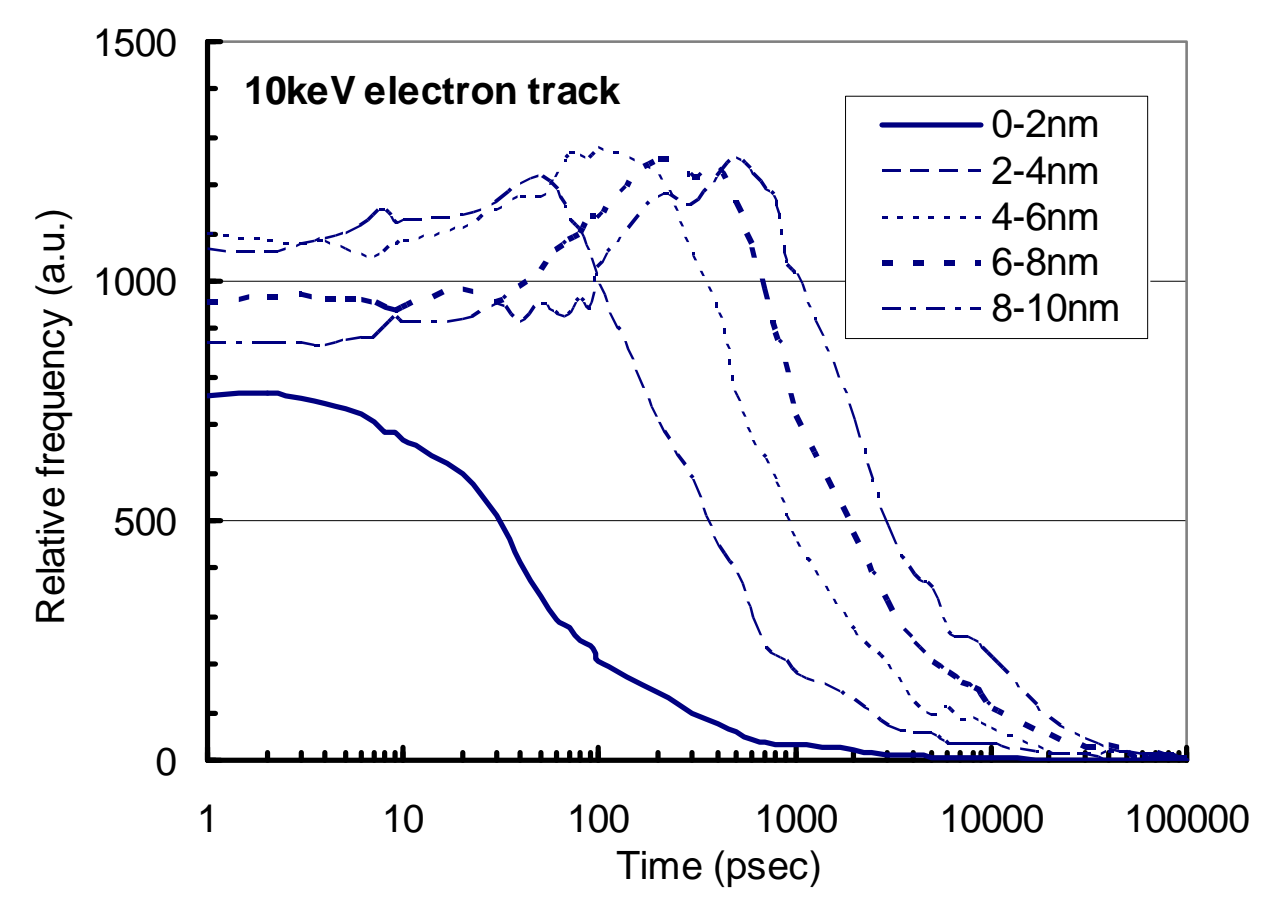

(a)

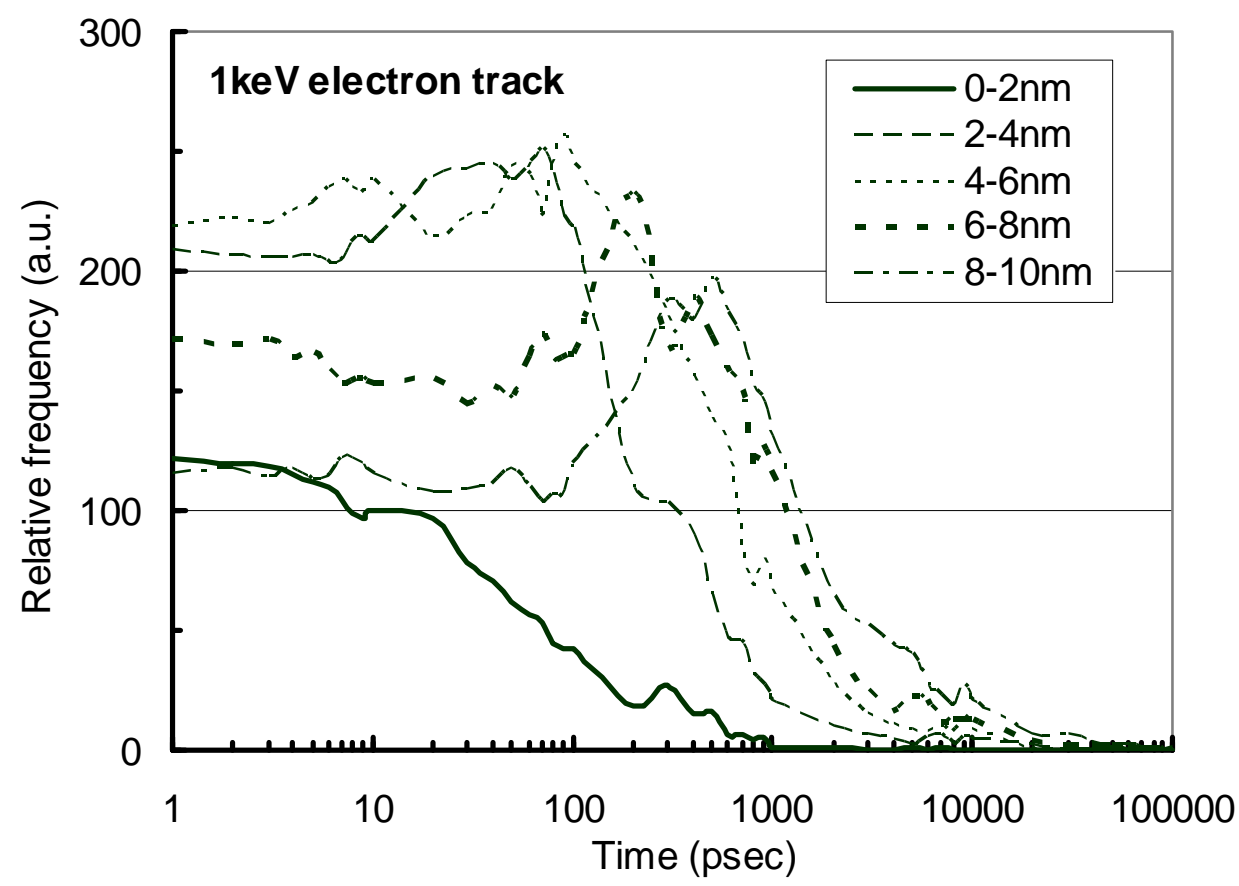

(b)

Figure 4.

Date et al. 